\title{
LITERATUR
}

\section{Parteien in Europa - Europäisierung der Parteien}

\author{
Uwe Jun*
}

\section{Begrenzte Europäisierungstendenzen}

Die Ein- und Auswirkungen des Prozesses der europäischen Integration auf nationalstaatliche Organisationen, Institutionen und politische Systeme in Europa insgesamt ist ein in den letzten Jahren expandierendes Forschungsfeld. In diesem Zusammenhang fällt häufig der Begriff der ,Europäisierung ', der in unterschiedlicher Weise in der Politikwissenschaft definiert und diskutiert wird. Der Begriff verweist nicht nur auf die Konstruktion, Verbreitung und Institutionalisierung von formalen und informalen Regeln, Verfahren, Paradigmen, Handlungen sowie geteilten Werten und Normen, die von der Ebene der Europäischen Union ausgehend in nationalstaatliche Diskurse, Strukturen und Politiken Europas einfließen. ${ }^{1}$ Er umfasst bei einzelnen Autoren auch den Prozess der Institutionalisierung des politischen Systems der Europäischen Union. ${ }^{2}$

Thomas Poguntke, Nicholas Aylott, Elisabeth Carter, Robert Ladrech und Kurt Richard Luther favorisieren in ihrem Sammelband zum aktuellen Forschungsstand der Europäisierung nationalstaatlich verfasster Parteien in westeuropäischen Demokratien die erste Variante und konzeptionalisieren den Begriff als inner-organisatorischen Wandel politischer Parteien infolge des fortwährenden Prozesses der europäischen Integration. Der Wandel der
Thomas Poguntke/Nicholas Aylott/Elisabeth Carter/Robert Ladrech/Kurt Richard Luther (Hrsg.): The Europeanization of National Political Parties. Power and organizational adaptation, Routledge: London/New York 2007, ISBN 978-0-415-40191-3; 238 Seiten, 101,00 €.

Jürgen Mittag (Hrsg.): Politische Parteien und europäische Integration. Entwicklung und Perspektiven transnationaler Parteienkooperation in Europa, Klartext: Essen 2006, ISBN 3-89861702-5; 810 Seiten, 79,00€.

Parteien in Deutschland, Frankreich, Großbritannien, Österreich und Schweden ist in ihrem Untersuchungsdesign also die abhängige Variable. Konkret untersucht wird, ob sich infolge der europäischen Integration formale und informale Wandlungsprozesse in den Parteien ausmachen lassen und ob diese zu innerparteilichen Verschiebungen der Machtkonstellation geführt haben. Im Sinne theoretischer Konzepte zum Parteienwandel wird somit der Integrationsprozess als externer Stimuli zur organisatorischen Veränderung begriffen.

Im Vergleich zu Parlamenten, Regierungen oder den politischen Systemen insgesamt sind Europäisierungstendenzen politischer Parteien ein weniger bearbeitetes Feld. Daher ist dieser Band als Teil eines langfristig angelegten Projektes zu verstehen. Poguntke und sei-

* Prof. Dr. Uwe Jun, Geschäftsführer des Faches Politikwissenschaft, Universität Trier.

1 Claudio M. Radaelli: The Europeanization of Public Policy, in: Kevin Featherstone/Claudio M. Radaelli (Hrsg.): The Politics of Europeanization, Oxford 2003, S. 27-56.

2 Siehe Thomas Risse: A European Identity? Europeanization and the Evolution of Nation-State Identities, in: Maria Green Cowles/James A. Caporaso/Thomas Risse (Hrsg.): Transforming Europe: Europeanization and Domestic Change, Ithaca 2001, S. 198-216. 
nen Mitherausgebern wie auch den Autoren der einzelnen Beiträge gelingt es in erheblichem Maße das Feld zu bestellen und eine recht ertragreiche Ernte einzufahren. Dank der kohärenten Anlage des Bandes und der systematischen Herangehensweise bekommt der Leser ein klares und fundiertes Bild. Enthusiasten des europäischen Integrationsprozesses werden die Ergebnisse mit einiger Enttäuschung zur Kenntnis nehmen: Es kann nur sehr begrenzt von einer Europäisierung politischer Parteien in Westeuropa gesprochen werden. Die von den Herausgebern als zentral für Europäisierungstendenzen herausgearbeiteten Indikatoren lassen sich kaum finden. Weder ist eine deutliche Vermehrung von Experten für Angelegenheiten der Europäischen Union innerhalb der Parteien zu finden, noch lassen sich spürbare machtpolitische oder programmatische Einflussnahmen der EU-Ebene auf die Organisation beziehungsweise Ausrichtung der einzelnen Parteien konstatieren. Wie Nicholas Aylott, Laura Morales und Luis Ramiro in ihrer Zusammenfassung der Ergebnisse der einzelnen Länderkapitel hervorheben, findet die europäische Integration bei den Parteien durchaus mehr Beachtung und Aufmerksamkeit, ohne sich aber in einem größeren organisatorischen Wandel niederzuschlagen.

\section{Autonomiegewinne für Kabinettsmitglieder}

Eine in der Parteienforschung allgemeiner zu findende Tendenz wird auch von der Forschergruppe dieses Bandes bestätigt, nämlich dass die Parteiführungen an Autonomie dazu gewonnen haben, in diesem Fall primär die Parteieliten der Regierungsparteien durch ihre Mitentscheidungsmacht im Ministerrat beziehungsweise im Europäischen Rat. Thomas Poguntke verweist in seinem Länderbeitrag zu Deutschland darauf, dass das Handeln der nationalen Kabinettsmitglieder in den europäischen Institutionen weder von den Parteien noch vom Bundestag hinreichend kontrolliert wird, was zu einer Verstärkung des Demokratiedefizits der Europäischen Union beiträgt. Selbst die Abgeordneten im Europäischen
Parlament unterliegen kaum einer Kontrolle durch ihre nationale Partei beziehungsweise regionale Parteigliederung, obwohl diese doch für die Rekrutierung der Mandatsträger in Brüssel und Straßburg verantwortlich zeichnen. Der Grund ist schnell gefunden: Es herrscht in den Parteien schlichtweg nur geringes Interesse an der Politik auf europäischer Ebene. Lediglich in den Fällen, in denen die Auswirkungen auf die Politik im Nationalstaat innerparteilich kontrovers diskutiert werden, ist eine größere innerparteiliche Aufmerksamkeit zu erkennen und dann wirken die EU-Experten (Parlamentarier, Mitarbeiter auf Fraktions- und Parteiebene) als eine Art ,Frühwarnsystem". Aber, wie Robert Ladrech verdeutlicht, ,once an EU-issue has entered the domestic arena, the usefulness of EU specialists, including MEPs, is almost nil, as the matter has now become interwoven into regular party management responsibilities" (S. 224). In Deutschland versuchen die Abgeordneten des Europäischen Parlaments mit einer Angebotsstrategie dem Desinteresse an europäischen Themen entgegenzuwirken und politischen Einfluss auf nationaler Ebene zu gewinnen, indem sie ihre vermehrten Kompetenzen auf europäischer Ebene gegen innerparteiliche Machtanteile eintauschen möchten, das heißt, dass sie ihre in erheblichem Ausmaß vorhandene Autonomie auf europäischer Ebene partiell abgeben und ihrer jeweiligen Partei vermehrt Einfluss auf ihre Entscheidungen im Parlament einräumen. Damit wiederum berauben sie sich partiell eines Vorteils ihrer Stellung im Gesamtgefüge der drei Ebenen Europäische Union, Nationalstaat und Wahlkreis.

\section{Schwache transnationale Parteibünde}

Dass die Mitglieder des Europäischen Parlaments Machtgewinne in ihrer nationalstaatlich verfassten Partei anstreben, kann zu erheblichen Teilen mit der sehr geringen machtpolitischen Relevanz der transnationalen Parteienbünde erklärt werden, die ihnen diesbezüglich keinen attraktiven Handlungsraum bieten. Transnationale Parteienbünde 
fristen nicht nur realpolitisch, sondern auch in der Politikwissenschaft eher ein Schattendasein. Schon allein aus diesem Grunde ist es verdienstvoll, dass sich Jürgen Mittag mit seiner Edition umfassend diesem Thema zugewandt hat. In 32 Abhandlungen und auf mehr als 700 Seiten behandelt der Sammelband nahezu alle Facetten der Entwicklung transnationaler Parteienkooperation in Europa. Mittags Buch will dazu beitragen, das lange Zeit ungelöste Problem der wissenschaftlichen Einordnung und Bewertung der Parteienbünde zu lösen. Dieser Versuch gelingt recht weitgehend, in dem historische, theoretisch-konzeptionelle und programmatische Aspekte des Handelns der Parteienbünde ebenso in den Blick genommen werden wie Rahmenbedingungen und Handlungsfelder. Auch wenn der Band über den aktuellen Forschungsstand nicht hinausgeht und auch dieser in der Einleitung nicht ganz lückenlos aufgearbeitet wird (Mittag übersieht darin, dass schon Mitte der Neunzigerjahre einzelne Monographien zum Thema vorlagen), so kann der Leser allerlei Erhellendes, Wissenswertes und Anregendes für zukünftige Forschungsarbeiten finden. Der Herausgeber selbst bietet dazu eine gelungene Einleitung und gemeinsam mit Eva Bräth eine Zusammenfassung der Beiträge an. Ihr Fazit überrascht kaum, wenn sie schreiben, dass „eine ernüchternde Bilanz der Legitimationsleistung transnationaler Parteizusammenarbeit" (S. 713) gezogen werden kann. Es ließe sich ergänzen, dass nach dem Lesen des Buches die Ernüchterung über die Arbeit der transnationalen Parteienkooperationen in Europa weit über Legitimationsaspekte hinausgeht. Die Parteienbünde haben bislang ihr Potenzial mit Blick auf ihre Einwirkung auf Politikprozesse auf europäischer oder globaler Ebene bei Weitem nicht ausschöpfen können. Ernst Kuper gibt in einem der lesenswertesten Beiträge des Bandes eine Erklärung für diesen Sachverhalt: es fehlt sowohl an einer europäischen Identität wie an einer europäischen Öffentlichkeit, was als Voraussetzung für einen erheblichen Bedeutungsgewinn der transnationalen Parteienkooperationen gesehen werden kann. Etwa las- sen sich seit der ersten Direktwahl des Europäischen Parlaments kleine Terraingewinne der Parteienbünde erkennen, insbesondere - wie Torsten Oppelland plausibel verdeutlicht - mit Blick auf die Fraktionen im Parlament, doch sind die Gewinne noch gering ausgeprägt.

\section{Ausblick: engere Verbindung von Integrati- ons- und Parteienforschung}

Wer nach Gründen für den geringen Europäisierungsgrad der nach wie vor stark nationalstaatlich orientierten politischen Parteien und für die recht geringe Relevanz der europäischen Parteienbünde in beiden Sammelbänden sucht, der wird an einzelnen Stellen immer wieder etwas finden, ein systematisches Fazit oder eine mit den Ergebnissen der Parteienforschung verbundene Synthese jedoch letztlich vermissen. So weist Mittag in seiner Einleitung zutreffend darauf hin, dass die europäischen Parteiorganisationen weder bei der Vergabe zentraler Ämter auf europäischer Ebene noch bei der Rekrutierung der Abgeordneten des Europäischen Parlaments eine nennenswerte Rolle spielen (S. 26). Ladrech bekräftigt im abschließenden Beitrag des von Poguntke et al. herausgegebenen Buches diese Beobachtung und weist auf den geringen programmatischen Einfluss der Parteienbünde auf das Europäische Parlament oder die einzelnen Mitgliedsparteien hin. An diesem Punkt der Forschung müsste weiter gearbeitet werden. Der Prozess der Europäisierung oder die transnationalen Parteienbünde spielen letztlich für die primär auf nationalstaatlicher Ebene agierenden und organisierten Parteien keine nennenswerte Rolle, weder mit Blick auf deren Organisationsstruktur noch auf deren Ziele. Auf der Ebene der einfachen Mitglieder (,,party on the ground") spiegelt sich de Relevanzgewinn der Europäischen Union kaum wider, für die ,party in public office“ (Amts- und Mandatsträger) oder die „party in central office“ (Parteiführungen, Parteizentrale) lassen sich kaum Anreize für eine stärkere Berücksichtigung des Integrationsprozesses im Organisationsleben oder gar eine 
Hinwendung zu transnationalen Parteibünden ausmachen. Im Gegenteil: Die Dichotomie von politischen und ökonomischen Eliten einerseits und Teilen der Bevölkerung andererseits in Fragen der europäischen Einigung, wie sie in Referenden immer mal wieder zutage tritt, lässt sich auch auf die Organisation zumindest einiger Parteien übertragen, das heißt in vielen Parteien sind nicht selten skeptische Töne der Mitgliedschaft vernehmbar, selbst wenn die Führung grundsätzlich proeuropäisch ausgerichtet ist. Auch für die Ziele von politischen Parteien wie Stimmenmaximierung oder die Erlangung öffentlicher Ämter spielt die Ebene der Europäischen Union für Parteien eine sehr untergeordnete Rolle.
Sie müssen Wahlen auf der Ebene des Nationalstaats oder der Region gewinnen und besetzen hauptsächlich auf diesen Ebenen zu vergebende Ämter und Mandate. Solange in dieser Hinsicht keine Änderungen eintreten, wird die Forschung wohl auch in Zukunft feststellen, dass die Europäisierung nationalstaatlich verfasster Parteien und der Bedeutungsgewinn transnationaler Parteienbünde kaum vorankommt. Die Dynamik der europäischen Integration wie eine engere Verbindung von Integrations- und Parteienforschung bietet dem Forschungsgebiet aber genügend Stoff für weitere interessante Projekte und $\mathrm{Pu}$ blikationen wie diese.

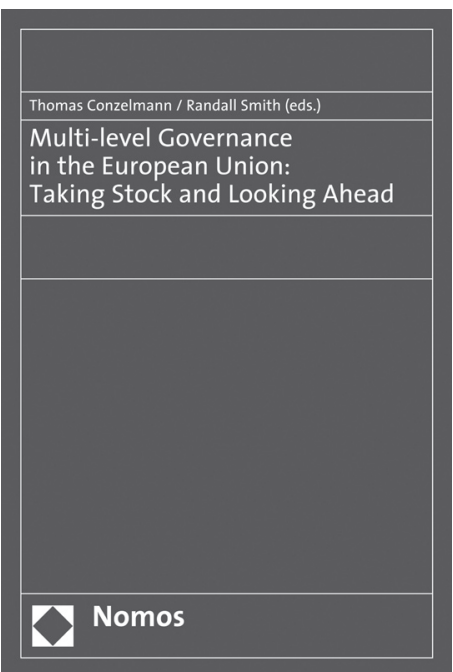

\section{Multi-Level Governance in the European Union: Taking Stock and Looking Ahead} Herausgegeben von Prof. Dr. phil. Thomas Conzelmann und Dr. Randall Smith 2008, 269 S., brosch., 39,- $€$, ISBN 978-3-8329-3838-3

Der Band beinhaltet eine kritische Bestandsaufnahme der Governance-Debatten der letzten Jahre hinsichtlich der Effektivität und der demokratischen Legitimität verschiedener Formen von Governance. Die Beiträge erweitern zudem anhand von Fallbeispielen den bisherigen Horizont der Diskussion und betreten sowohl in empirischer als auch in theoretischer Hinsicht Neuland. 